\title{
Palladium-Catalyzed Formal [4+2] Cycloaddtion of $o$-Xylylenes with Olefins
}

\author{
Ryoichi Kuwano* and Takenori Shige \\ Department of Chemistry, Graduate School of Sciences, Kyushu University, 6-10-1 Hakozaki, Higashi-ku, \\ Fukuoka 812-8581, Japan
}

\section{Supporting Information}

General and Materials. NMR spectra were measured with Bruker AVANCE 400 (9.4 T magnet) spectrometer. IR spectra were measured with Nicolet Magna 560 or JASCO FT/IR-4100. Elemental analyses were performed by Service Centre of Elementary Analysis of Organic Compounds in Kyushu University. Flash column chromatographies and medium-pressure liquid chromatographies (MPLC) were performed with silica gel 60 (230-400 mesh, Merck) and C.I.G. pre-packed column CPS-223L-1 (Kusano, Tokyo, Japan), respectively.

Dichloromethane $\left(\mathrm{CH}_{2} \mathrm{Cl}_{2}\right)$, pyridine, $N, N, N^{\prime}, N^{\prime}$-tetramethylethylenediamine (TMEDA), and dimethylsulfoxide (DMSO) were dried with calcium hydride. Diethyl ether $\left(\mathrm{Et}_{2} \mathrm{O}\right)$ was dried with sodium-benzophenone ketyl. These solvents and reagents were distilled under nitrogen atmosphere. THF (HPLC grade, without inhibitor) was deoxidized by purging with nitrogen for 30 min and was dried with an alumina column system (GlassContour Co.). 2-[(Trimethylsilyl)methyl]benzyl alcohol, ${ }^{1}$ 4-methoxy-2-methylbenzyl alcohol, ${ }^{2}$ and $\operatorname{Pd}\left(\eta^{3}-\mathrm{C}_{3} \mathrm{H}_{5}\right) \mathrm{Cp}^{3,4}$ were prepared according to literature procedures. DPPE was purchased from KANTO CHEMICAL. All other materials were commercially available. Liquid olefins 2 were distilled at appropriate pressure before use. Other purchased compounds were used without further purification.

\section{Preparations of $o$-Xylylene Precursors 1.}

\section{Methyl 2-[(Trimethylsilyl)methyl]benzyl Carbonate (1a) [909792-50-3]. ${ }^{5}$}

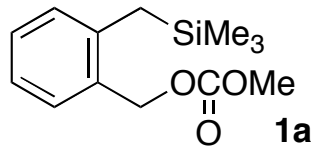

A solution of methyl chloroformate $(1.8 \mathrm{ml}, d 1.223 \mathrm{~g} / \mathrm{ml}, 23 \mathrm{mmol})$ in dry $\mathrm{CH}_{2} \mathrm{Cl}_{2}(5.0 \mathrm{ml})$ was added dropwise to a solution of 2-[(trimethylsilyl)methyl]benzyl alcohol (4.00 g, $21 \mathrm{mmol})$ and pyridine $(2.0 \mathrm{ml}, d$ $0.978 \mathrm{~g} / \mathrm{ml}, 25 \mathrm{mmol})$ in $\mathrm{CH}_{2} \mathrm{Cl}_{2}(20 \mathrm{ml})$ at $0{ }^{\circ} \mathrm{C}$ for $45 \mathrm{~min}$. The mixture was stirred at room temperature for $1 \mathrm{~h}$. After $1 \mathrm{~N} \mathrm{HCl} \mathrm{aq.} \mathrm{was} \mathrm{added} \mathrm{to} \mathrm{the} \mathrm{resulting} \mathrm{mixture,} \mathrm{the} \mathrm{organic} \mathrm{layer} \mathrm{was} \mathrm{separated.} \mathrm{The}$ aqueous layer was extracted twice with EtOAc. The combined organic layer was washed with brine, was dried with $\mathrm{MgSO}_{4}$, and then was evaporated under reduced pressure. The residue was purified with a flash column chromatography $($ EtOAc/hexane $=1 / 5)$ to give $\mathbf{1 a}(5.13 \mathrm{~g}, 99 \%)$ as colorless oil: ${ }^{1} \mathrm{H} \mathrm{NMR}(400 \mathrm{MHz}$, 
$\left.\mathrm{CDCl}_{3}, \mathrm{TMS}\right) \delta 0.01(\mathrm{~s}, 9 \mathrm{H}), 2.19(\mathrm{~s}, 2 \mathrm{H}), 3.79(\mathrm{~s}, 3 \mathrm{H}), 5.14(\mathrm{~s}, 2 \mathrm{H}), 7.02(\mathrm{~d}, J=7.6 \mathrm{~Hz}, 1 \mathrm{H}), 7.09(\mathrm{dt}, J=$ $0.8,7.4 \mathrm{~Hz}, 1 \mathrm{H}), 7.21(\mathrm{dt}, J=1.3,7.5 \mathrm{~Hz}, 1 \mathrm{H}), 7.33(\mathrm{~d}, J=7.5 \mathrm{~Hz}, 1 \mathrm{H}) ;{ }^{13} \mathrm{C}\left\{{ }^{1} \mathrm{H}\right\} \mathrm{NMR}\left(100 \mathrm{MHz}, \mathrm{CDCl}_{3}\right)$ $\delta-1.5,23.1,54.8,68.1,124.3,128.5,129.3,129.8,131.5,139.7,155.8$; IR (neat) 2956, 1750, $1269 \mathrm{~cm}^{-1}$; Anal. Calcd for $\mathrm{C}_{13} \mathrm{H}_{20} \mathrm{O}_{3} \mathrm{Si}$ : C, 61.87; H, 7.99. Found: C, 62.08; H, 8.01.

\section{4-Methoxy-2-[(trimethylsilyl)methyl]benzyl Alcohol.}

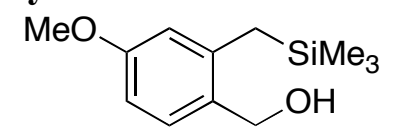

A solution of butyllithium in hexane $(1.6 \mathrm{M}, 9.6 \mathrm{ml}, 15 \mathrm{mmol})$ was added dropwise to a solution of 4-methoxy-2-methylbenzyl alcohol (1.00 g, $6.6 \mathrm{mmol})$ and TMEDA (2.8 ml, $d 0.775 \mathrm{~g} / \mathrm{ml}, 19 \mathrm{mmol})$ in dry $\mathrm{Et}_{2} \mathrm{O}(13 \mathrm{ml})$ at $0{ }^{\circ} \mathrm{C}$ for $1 \mathrm{~h}$. After stirred under reflux for $24 \mathrm{~h}$, the reaction mixture was cooled to $-78^{\circ} \mathrm{C}$. Chlorotrimethylsilane $(2.3 \mathrm{ml}, d 0.856 \mathrm{~g} / \mathrm{ml}, 18 \mathrm{mmol})$ was added dropwise to the resulting solution for 40 min. The mixture was stirred at room temperature for $3 \mathrm{~h}$, and then $10 \% \mathrm{H}_{2} \mathrm{SO}_{4}$ aq. (7.3 ml) was added dropwise for $1 \mathrm{~h}$. After stirred for $20 \mathrm{~h}$, the mixture was extract five times with EtOAc. The combined organic layer was washed with brine, was dried with $\mathrm{Na}_{2} \mathrm{SO}_{4}$, and then was evaporated under reduced pressure. The residue was passed through a flash column chromatography (EtOAc/hexane $=1 / 15)$. The fraction containing the desired product was evaporated, and then was purified with MPLC (EtOAc/hexane = $1 / 1$ ) to give the desired silylated product (502 mg, 34\%), which contained a small amount of impurities. We used the mixture for the preparation of $\mathbf{1 b}$ without further purification. Colorless oil: ${ }^{1} \mathrm{H}$ NMR (400 MHz, $\left.\mathrm{CDCl}_{3}, \mathrm{TMS}\right) \delta 0.02(\mathrm{~s}, 9 \mathrm{H}), 1.31$ (br t, $\left.J=5.0 \mathrm{~Hz}, 1 \mathrm{H}\right), 2.20$ (s, 2H), 3.78 (s, 3H), 4.59 (br d, $J=5.0 \mathrm{~Hz}$, $2 \mathrm{H}), 6.57(\mathrm{~d}, J=2.7 \mathrm{~Hz}, 1 \mathrm{H}), 6.64(\mathrm{dd}, J=2.7,8.4 \mathrm{~Hz}, 1 \mathrm{H}), 7.22(\mathrm{~d}, J=8.4 \mathrm{~Hz}, 1 \mathrm{H}) ;{ }^{13} \mathrm{C}\left\{{ }^{1} \mathrm{H}\right\} \mathrm{NMR}(100$ $\left.\mathrm{MHz}, \mathrm{CDCl}_{3}\right) \delta-1.4,23.3,55.1,63.4,109.6,114.9,129.8,130.1,140.7,159.1$.

\section{4-Methoxy-2-[(trimethylsilyl)methyl]benzyl Methyl Carbonate (1b).}

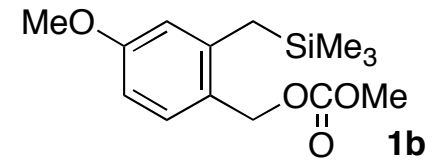

A solution of methyl chloroformate $(0.15 \mathrm{ml}, d 1.223 \mathrm{~g} / \mathrm{ml}, 1.9 \mathrm{mmol})$ in dry $\mathrm{CH}_{2} \mathrm{Cl}_{2}(0.5 \mathrm{ml})$ was added dropwise to a solution of 4-methoxy-2-[(trimethylsilyl)methyl]benzyl alcohol (346 mg, $1.5 \mathrm{mmol})$, pyridine $(0.15 \mathrm{ml}, d 0.978 \mathrm{~g} / \mathrm{ml}, 1.9 \mathrm{mmol})$, and 4-(dimethylamino)pyridine $(1.7 \mathrm{mg}, 14 \mu \mathrm{mol})$ in $\mathrm{CH}_{2} \mathrm{Cl}_{2}(1.0 \mathrm{ml})$ at $0{ }^{\circ} \mathrm{C}$ for $20 \mathrm{~min}$. The mixture was stirred at room temperature for $1 \mathrm{~h}$. After $1 \mathrm{~N} \mathrm{HCl} \mathrm{aq}$. was added, the resulting mixture was extracted three times with EtOAc. The combined organic layer was washed with brine, was dried with $\mathrm{Na}_{2} \mathrm{SO}_{4}$, and then was evaporated under reduced pressure. The residue was purified with a flash column chromatography $($ EtOAc/hexane $=1 / 10)$ to give $\mathbf{1 b}(412 \mathrm{mg}, 95 \%)$ as colorless oil: ${ }^{1} \mathrm{H}$ NMR (400 MHz, $\mathrm{CDCl}_{3}$, TMS) $\delta 0.02(\mathrm{~s}, 9 \mathrm{H}), 2.18$ (s, 2H), 3.775 (s, 3H), 3.777 (s, 3H), 5.07 (s, $\left.2 \mathrm{H}\right), 6.56$ $(\mathrm{d}, J=2.7 \mathrm{~Hz}, 1 \mathrm{H}), 6.63(\mathrm{dd}, J=2.7,8.3 \mathrm{~Hz}, 1 \mathrm{H}), 7.25(\mathrm{~d}, J=8.3 \mathrm{~Hz}, 1 \mathrm{H}) ;{ }^{13} \mathrm{C}\left\{{ }^{1} \mathrm{H}\right\} \mathrm{NMR}(100 \mathrm{MHz}$, 
$\left.\mathrm{CDCl}_{3}\right) \delta-1.5,23.5,54.7,55.1,68.0,109.6,114.7,124.1,131.9,141.9,155.8,159.7$; IR (neat) 2956, 1747, $1255 \mathrm{~cm}^{-1}$; Anal. Calcd for $\mathrm{C}_{14} \mathrm{H}_{22} \mathrm{O}_{4} \mathrm{Si}: \mathrm{C}, 59.54 ; \mathrm{H}, 7.85$. Found: C, 59.77; H, 7.87.

\section{4-Methyl-2-[(trimethylsilyl)methyl]benzyl Alcohol.}

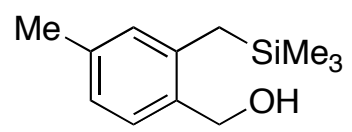

A solution of butyllithium in hexane $(1.6 \mathrm{M}, 10.5 \mathrm{ml}, 17 \mathrm{mmol})$ was added dropwise to a solution of 2,4-dimethylbenzyl alcohol (1.11 g, $8.2 \mathrm{mmol})$ and TMEDA (3.0 ml, $d 0.775 \mathrm{~g} / \mathrm{ml}, 20 \mathrm{mmol})$ in dry THF (14 $\mathrm{ml}$ ) at $-20^{\circ} \mathrm{C}$ for $1 \mathrm{~h}$. After stirred at room temperature for $3 \mathrm{~h}$, the reaction mixture was cooled to $-78{ }^{\circ} \mathrm{C}$. Chlorotrimethylsilane $(2.5 \mathrm{ml}, d 0.856 \mathrm{~g} / \mathrm{ml}, 20 \mathrm{mmol})$ was added dropwise to the resulting solution for 40 min. The mixture was stirred at room temperature for $7 \mathrm{~h}$, and then $10 \% \mathrm{H}_{2} \mathrm{SO}_{4} a q$. (40 ml) was added dropwise for $20 \mathrm{~min}$. After stirred for $15 \mathrm{~h}$, the mixture was extract three times with EtOAc. The combined organic layer was washed with brine, was dried with $\mathrm{Na}_{2} \mathrm{SO}_{4}$, and then was evaporated under reduced pressure. The residue was purified with a flash column chromatography $($ EtOAc/hexane $=1 / 5)$ to give the starting alcohol $(594 \mathrm{mg}, 53 \%)$ as well as the desired silylated product (466 $\mathrm{mg}, 27 \%$ ), which contained a small amount of impurities. We used the mixture for the preparation of 1c without further purification. Yellow oil: ${ }^{1} \mathrm{H}$ NMR $\left(400 \mathrm{MHz}, \mathrm{CDCl}_{3}\right.$, TMS) $\delta 0.00$ (s, 9H), 1.38 (br, 1H), 2.16 (s, $\left.2 \mathrm{H}\right), 2.29$ $(\mathrm{s}, 3 \mathrm{H}), 4.59(\mathrm{~d}, J=4.5 \mathrm{~Hz}, 2 \mathrm{H}), 6.83(\mathrm{~s}, 1 \mathrm{H}), 6.90(\mathrm{~d}, J=7.7 \mathrm{~Hz}, 1 \mathrm{H}), 7.20(\mathrm{~d}, J=7.7 \mathrm{~Hz}, 1 \mathrm{H}) ;{ }^{13} \mathrm{C}\left\{{ }^{1} \mathrm{H}\right\}$ $\operatorname{NMR}\left(100 \mathrm{MHz}, \mathrm{CDCl}_{3}\right) \delta-1.4,21.2,22.8,63.5,125.2,128.5,130.1,134.2,137.3,138.7$.

\section{Methyl 4-Methyl-2-[(trimethylsilyl)methyl]benzyl Carbonate (1c).

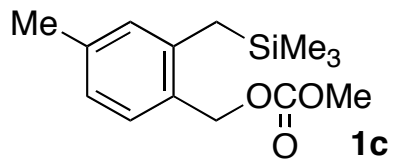

A solution of methyl chloroformate $(80 \mu \mathrm{l}, d 1.223 \mathrm{~g} / \mathrm{ml}, 1.0 \mathrm{mmol})$ in dry $\mathrm{CH}_{2} \mathrm{Cl}_{2}(0.1 \mathrm{ml})$ was added dropwise to a solution of 4-methyl-2-[(trimethylsilyl)methyl]benzyl alcohol (204 mg, $0.98 \mathrm{mmol})$, pyridine $(0.10 \mathrm{ml}, d 0.978 \mathrm{~g} / \mathrm{ml}, 1.2 \mathrm{mmol})$, and 4-(dimethylamino)pyridine $(2.6 \mathrm{mg}, 21 \mu \mathrm{mol})$ in $\mathrm{CH}_{2} \mathrm{Cl}_{2}(0.9 \mathrm{ml})$ at $0{ }^{\circ} \mathrm{C}$ for $1 \mathrm{~h}$. The mixture was stirred at room temperature for $1 \mathrm{~h}$. After water was added, the resulting mixture was extracted three times with EtOAc. The combined organic layer was washed with brine, was dried with $\mathrm{Na}_{2} \mathrm{SO}_{4}$, and then was evaporated under reduced pressure. The residue was purified with a flash column chromatography $($ EtOAc/hexane $=1 / 5)$ to give $1 \mathrm{c}(246 \mathrm{mg}, 94 \%)$ as colorless oil: ${ }^{1} \mathrm{H}$ NMR (400 $\left.\mathrm{MHz}, \mathrm{CDCl}_{3}, \mathrm{TMS}\right) \delta 0.00(\mathrm{~s}, 9 \mathrm{H}), 2.15$ (s, 2H), 2.29 (s, 3H), 3.78 (s, 3H), 5.09 (s, 2H), $6.83(\mathrm{~s}, 1 \mathrm{H}), 6.89(\mathrm{~d}$, $J=7.7 \mathrm{~Hz}, 1 \mathrm{H}), 7.21(\mathrm{~d}, J=7.7 \mathrm{~Hz}, 1 \mathrm{H}) ;{ }^{13} \mathrm{C}\left\{{ }^{1} \mathrm{H}\right\} \mathrm{NMR}\left(100 \mathrm{MHz}, \mathrm{CDCl}_{3}\right) \delta-1.5,21.2,23.0,54.7,68.0$, 125.1, 128.6, 130.0, 130.1, 138.3, 139.7, 155.8; IR (neat) 2952, 1746, $1269 \mathrm{~cm}^{-1}$; Anal. Calcd for $\mathrm{C}_{14} \mathrm{H}_{22} \mathrm{O}_{3} \mathrm{Si}: \mathrm{C}, 63.12 ; \mathrm{H}, 8.32$. Found: C, 63.32; H, 8.29. 


\section{3-Phenyl-2-[(trimethylsilyl)methyl]benzyl Alcohol [909792-54-7].}<smiles>CCc1c(CO)cccc1-c1ccccc1</smiles>

A solution of butyllithium in hexane $(1.6 \mathrm{M}, 26 \mathrm{ml}, 42 \mathrm{mmol})$ was added dropwise to a solution of 2-methyl-3-phenylbenzyl alcohol $(3.98 \mathrm{~g}, 20 \mathrm{mmol})$ and TMEDA $(6.3 \mathrm{ml}, d 0.775 \mathrm{~g} / \mathrm{ml}, 42 \mathrm{mmol})$ in dry THF $(13 \mathrm{ml})$ at $0{ }^{\circ} \mathrm{C}$ for $40 \mathrm{~min}$. After stirred at room temperature for $24 \mathrm{~h}$, the reaction mixture was cooled to $-78{ }^{\circ} \mathrm{C}$. Chlorotrimethylsilane $(6.3 \mathrm{ml}, d 0.856 \mathrm{~g} / \mathrm{ml}, 50 \mathrm{mmol})$ was added dropwise to the resulting solution for $1 \mathrm{~h}$. The mixture was stirred at room temperature for $5 \mathrm{~h}$, and then $10 \% \mathrm{H}_{2} \mathrm{SO}_{4} a q$. (60 ml) was added dropwise for $1 \mathrm{~h}$. After stirred for $16 \mathrm{~h}$, the mixture was extract three times with EtOAc. The combined organic layer was washed with brine, was dried with $\mathrm{Na}_{2} \mathrm{SO}_{4}$, and then was evaporated under reduced pressure. The residue was purified with a flash column chromatography $($ EtOAc/hexane $=1 / 3)$ to give the starting alcohol $(1.59 \mathrm{~g}, 40 \%)$ as well as the desired silylated product $(2.88 \mathrm{~g}, 53 \%)$, which contained a small amount of impurities. We used the mixture for the preparation of $\mathbf{1 d}$ without further purification. Yellow oil: ${ }^{1} \mathrm{H}$ NMR (400 MHz, $\left.\mathrm{CDCl}_{3}, \mathrm{TMS}\right) \delta-0.29(\mathrm{~s}, 9 \mathrm{H}), 1.51(\mathrm{t}, J=5.8 \mathrm{~Hz}, 1 \mathrm{H}), 2.38(\mathrm{~s}, 2 \mathrm{H}), 4.70(\mathrm{~d}$, $J=5.8 \mathrm{~Hz}, 2 \mathrm{H}), 7.10-7.17(\mathrm{~m}, 2 \mathrm{H}), 7.29-7.42(\mathrm{~m}, 6 \mathrm{H}) ;{ }^{13} \mathrm{C}\left\{{ }^{1} \mathrm{H}\right\} \mathrm{NMR}\left(100 \mathrm{MHz}, \mathrm{CDCl}_{3}\right) \delta-0.6,19.0,64.3$, $124.0,126.7,127.0,128.1,129.8,130.0,137.2,137.7,141.4,142.7$.

\section{Methyl 3-Phenyl-2-[(trimethylsilyl)methyl]benzyl Carbonate (1d).}

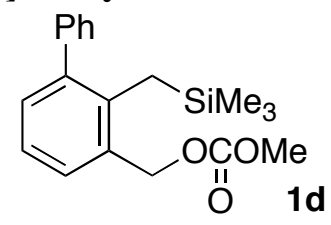

A solution of methyl chloroformate $(0.97 \mathrm{ml}, d 1.223 \mathrm{~g} / \mathrm{ml}, 1.0 \mathrm{mmol})$ in dry $\mathrm{CH}_{2} \mathrm{Cl}_{2}(2.0 \mathrm{ml})$ was added dropwise to a solution of 3-phenyl-2-[(trimethylsilyl)methyl]benzyl alcohol (2.60 g, $9.6 \mathrm{mmol})$, pyridine (0.99 ml, $d 0.978 \mathrm{~g} / \mathrm{ml}, 12 \mathrm{mmol})$, and 4-(dimethylamino)pyridine (12 $\mathrm{mg}, 99 \mu \mathrm{mol})$ in $\mathrm{CH}_{2} \mathrm{Cl}_{2}(8.0 \mathrm{ml})$ at $0{ }^{\circ} \mathrm{C}$ for $30 \mathrm{~min}$. The mixture was stirred at room temperature for $1 \mathrm{~h}$. After $1 \mathrm{~N} \mathrm{HCl} \mathrm{aq}$. was added, the resulting mixture was extracted twice with EtOAc. The combined organic layer was washed with brine, was dried with $\mathrm{Na}_{2} \mathrm{SO}_{4}$, and then was evaporated under reduced pressure. The residue was purified with a flash column chromatography $($ EtOAc/hexane $=1 / 10)$ to give 1d $(2.97 \mathrm{~g}, 94 \%)$ as a colorless solid: $\mathrm{mp}$ $57{ }^{\circ} \mathrm{C} ;{ }^{1} \mathrm{H}$ NMR $\left(400 \mathrm{MHz}, \mathrm{CDCl}_{3}\right.$, TMS) $\delta-0.29$ (s, 9H), 2.37 (s, 2H), 3.81 (s, 3H), 5.19 (s, 2H), 7.10-7.17 $(\mathrm{m}, 2 \mathrm{H}), 7.28-7.42(\mathrm{~m}, 6 \mathrm{H}) ;{ }^{13} \mathrm{C}\left\{{ }^{1} \mathrm{H}\right\} \mathrm{NMR}\left(100 \mathrm{MHz}, \mathrm{CDCl}_{3}\right) \delta-0.7,19.3,54.8,68.8,123.9,126.8,128.1$, $128.9,129.8,130.9,132.1,138.2,141.5,142.5,155.8$; IR (neat) 2955, 1749, $1262 \mathrm{~cm}^{-1}$; Anal. Calcd for $\mathrm{C}_{19} \mathrm{H}_{24} \mathrm{O}_{3} \mathrm{Si}: \mathrm{C}, 69.47 ; \mathrm{H}, 7.36$. Found: C, 69.51; H, 7.34. 


\section{4,6-Dimethyl-2-[(trimethylsilyl)methyl]benzyl Alcohol [909792-57-0].}

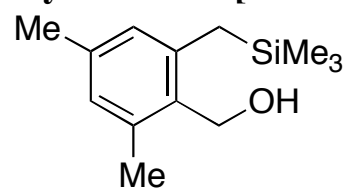

A solution of butyllithium in hexane $(1.6 \mathrm{M}, 2.8 \mathrm{ml}, 4.5 \mathrm{mmol})$ was added dropwise to a solution of 2,4,6-trimethylbenzyl alcohol $(301 \mathrm{mg}, 2.0 \mathrm{mmol})$ in dry $\mathrm{Et}_{2} \mathrm{O}(3.6 \mathrm{ml})$ at $0{ }^{\circ} \mathrm{C}$ for $30 \mathrm{~min}$. After stirred under reflux for $24 \mathrm{~h}$, the reaction mixture was cooled to $-78{ }^{\circ} \mathrm{C}$. Chlorotrimethylsilane $(0.64 \mathrm{ml}, d 0.856$ $\mathrm{g} / \mathrm{ml}, 5.0 \mathrm{mmol}$ ) was added dropwise to the resulting solution for $30 \mathrm{~min}$. The mixture was stirred at room temperature for $3 \mathrm{~h}$, and then $10 \% \mathrm{H}_{2} \mathrm{SO}_{4}$ aq. $(4 \mathrm{ml})$ and methanol $(1.5 \mathrm{ml})$ were added. After stirred for 16 $\mathrm{h}$, the mixture was diluted with water and was extracted three times with EtOAc. The combined organic layer was washed with brine, was dried with $\mathrm{Na}_{2} \mathrm{SO}_{4}$, and then was evaporated under reduced pressure. The residue was passed through a short silica gel column $($ EtOAc/hexane $=1 / 3)$, and then was purified with MPLC $(E t O A c / h e x a n e=1 / 3)$ to give the desired silylated product $(208 \mathrm{mg}, 47 \%)$, which contained a small amount of impurities. We used the mixture for the preparation of $1 \mathbf{e}$ without further purification. Yellow oil: ${ }^{1} \mathrm{H}$ NMR (400 MHz, CDCl, TMS) $\delta 0.00$ (s, 9H), 1.12 (br, 1H), $2.22(\mathrm{~s}, 2 \mathrm{H}), 2.25$ (s, 3H), 2.39 (s, 3H), $4.63(\mathrm{~s}, 2 \mathrm{H}), 6.69(\mathrm{~s}, 1 \mathrm{H}), 6.77(\mathrm{~s}, 1 \mathrm{H}) ;{ }^{13} \mathrm{C}\left\{{ }^{1} \mathrm{H}\right\} \mathrm{NMR}\left(100 \mathrm{MHz}, \mathrm{CDCl}_{3}\right) \delta-1.5,19.6,21.1,23.6,59.3$, $127.7,128.1,132.3,137.3,137.6,139.7$.

\section{Methyl 4,6-Dimethyl-2-[(trimethylsilyl)methyl]benzyl Carbonate (1e).}

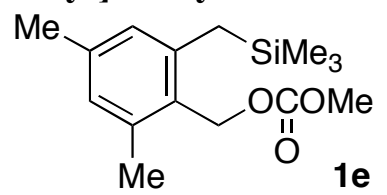

A solution of methyl chloroformate $(76 \mu \mathrm{l}, d 1.223 \mathrm{~g} / \mathrm{ml}, 0.98 \mathrm{mmol})$ in dry $\mathrm{CH}_{2} \mathrm{Cl}_{2}(0.1 \mathrm{ml})$ was added dropwise to a solution of 4,6-dimethyl-2-[(trimethylsilyl)methyl]benzyl alcohol (169 $\mathrm{mg}, 0.76 \mathrm{mmol})$ and pyridine $(74 \mu \mathrm{l}, d 0.978 \mathrm{~g} / \mathrm{ml}, 0.91 \mathrm{mmol})$ in $\mathrm{CH}_{2} \mathrm{Cl}_{2}(0.7 \mathrm{ml})$ at $0{ }^{\circ} \mathrm{C}$ for $30 \mathrm{~min}$. The mixture was stirred at room temperature for $1 \mathrm{~h}$. After $1 \mathrm{~N} \mathrm{HCl} a q$. was added, the resulting mixture was extracted three times with EtOAc. The combined organic layer was washed with brine, was dried with $\mathrm{MgSO}_{4}$, and then was evaporated under reduced pressure. The residue was purified with a flash column chromatography $($ EtOAc/hexane $=1 / 15)$ to give 1e $(192.8 \mathrm{mg}, 90 \%)$ as colorless oil: ${ }^{1} \mathrm{H}$ NMR $\left(400 \mathrm{MHz}, \mathrm{CDCl}_{3}, \mathrm{TMS}\right) \delta$ $-0.01(\mathrm{~s}, 9 \mathrm{H}), 2.21(\mathrm{~s}, 2 \mathrm{H}), 2.24(\mathrm{~s}, 3 \mathrm{H}), 2.35$ (s, 3H), 3.77 (s, 3H), $5.17(\mathrm{~s}, 2 \mathrm{H}), 6.69(\mathrm{~s}, 1 \mathrm{H}), 6.76(\mathrm{~s}, 1 \mathrm{H})$; ${ }^{13} \mathrm{C}\left\{{ }^{1} \mathrm{H}\right\}$ NMR $\left(100 \mathrm{MHz}, \mathrm{CDCl}_{3}\right) \delta-1.5,19.8,21.1,23.7,54.7,64.6,126.7,127.6,128.0,138.2,138.7$, 140.9, 156.0; IR (neat) 2957, 1744, $1270 \mathrm{~cm}^{-1}$; Anal. Calcd for $\mathrm{C}_{15} \mathrm{H}_{24} \mathrm{O}_{3} \mathrm{Si}$ : C, 64.24; H, 8.63. Found: $\mathrm{C}$, $64.37 ; \mathrm{H}, 8.53$.

\section{Palladium-Catalyzed Cyclization of 1 with Olefins (2).}

General Procedure for Palladium-Catalyzed Cyclization of 1 with 2. An $o$-xylylene precursor $1(0.50$ mmol) and olefin $2(0.60 \mathrm{mmol})$ was successively added to a solution of $\mathrm{Pd}\left(\eta^{3}-\mathrm{C}_{3} \mathrm{H}_{5}\right) \mathrm{Cp}(3.2 \mathrm{mg}, 15 \mu \mathrm{mol})$ 
and DPPE $(6.6 \mathrm{mg}, 17 \mu \mathrm{mol})$ in DMSO $(1.0 \mathrm{ml})$. After the solution was stirred at $120^{\circ} \mathrm{C}$ for $72 \mathrm{~h}($ or $48 \mathrm{~h})$, water was added. The mixture was extracted several times with hexane or EtOAc. The combined organic layer was washed with brine, was dried with $\mathrm{MgSO}_{4}$, and then was evaporated under reduced pressure. The residue was purified with MPLC (EtOAc/hexane) after passed through a short silica gel column. Alternatively, it was purified with a flash column chromatography (EtOAc/hexane).

\section{Methyl 1,2,3,4-Tetrahydronaphthalene-2-carboxylate (3a) [39246-30-5] (Table 1, entry 1). ${ }^{6}$

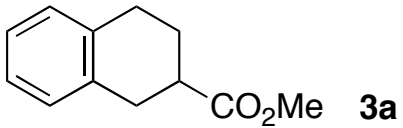

The general procedure was followed with 1a $(127 \mathrm{mg}, 0.50 \mathrm{mmol})$ and methyl acrylate (2a) $(52.8 \mathrm{mg}, 0.61$ mmol). The reaction was stopped at $48 \mathrm{~h}$. The crude product was purified with a flash column chromatography $($ EtOAc/hexane $=1 / 5)$ to give 3a $(74 \mathrm{mg}, 78 \%$ yield $)$ as colorless oil: ${ }^{1} \mathrm{H}$ NMR $(400 \mathrm{MHz}$, $\left.\mathrm{CDCl}_{3}, \mathrm{TMS}\right) \delta 1.86(\mathrm{ddt}, J=6.5,13.0,10.8 \mathrm{~Hz} 1 \mathrm{H}), 2.17-2.25(\mathrm{~m}, 1 \mathrm{H}), 2.70-2.79(\mathrm{~m}, 1 \mathrm{H}), 2.79-2.93(\mathrm{~m}$, 2H), 2.95-3.07 (m, 2H), 3.73 (s, 3H), 7.06-7.14 (m, 4H); ${ }^{13} \mathrm{C}\left\{{ }^{1} \mathrm{H}\right\}$ NMR (100 MHz, $\left.\mathrm{CDCl}_{3}\right) \delta 25.9,28.5$, $31.6,39.9,51.8,125.8,125.9,128.8,129.0,134.9,135.7,175.9$.

Methyl $\left(2 S^{*}, 3 S^{*}\right)-3-$ Methyl-1,2,3,4-tetrahydronaphthalene-2-carboxylate (3b) [112041-94-8] (Table 2, entry 1). ${ }^{7}$

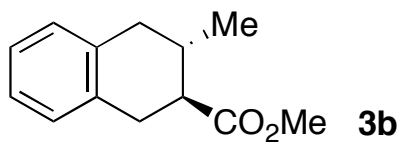

The general procedure was followed with $\mathbf{1 a}(126 \mathrm{mg}, 0.50 \mathrm{mmol})$ and methyl $(E)$-2-butenoate (2b) (60.4 $\mathrm{mg}, 0.60 \mathrm{mmol})$. The crude product was purified with MPLC (EtOAc/hexane $=1 / 5)$ to give $\mathbf{3 b}(59 \mathrm{mg}$, $58 \%$ yield) as yellow oil: ${ }^{1} \mathrm{H}$ NMR (400 MHz, $\mathrm{CDCl}_{3}$, TMS) $\delta 1.06(\mathrm{~d}, J=6.5 \mathrm{~Hz}, 3 \mathrm{H}), 2.04-2.20(\mathrm{~m}, 1 \mathrm{H})$, $2.45(\mathrm{dt}, J=5.3,10.9 \mathrm{~Hz}, 1 \mathrm{H}), 2.50(\mathrm{dd}, J=11.1,16.8 \mathrm{~Hz}, 1 \mathrm{H}), 2.89(\mathrm{dd}, J=5.1,16.8 \mathrm{~Hz}, 1 \mathrm{H}), 2.97(\mathrm{dd}, J=$ $5.3,16.5 \mathrm{~Hz}, 1 \mathrm{H}), 3.07(\mathrm{dd}, J=11.2,16.5 \mathrm{~Hz}, 1 \mathrm{H}), 3.73(\mathrm{~s}, 3 \mathrm{H}), 7.01-7.15(\mathrm{~m}, 4 \mathrm{H}) ;{ }^{13} \mathrm{C}\left\{{ }^{1} \mathrm{H}\right\} \mathrm{NMR}(100$ $\left.\mathrm{MHz}, \mathrm{CDCl}_{3}\right) \delta 19.8,31.7,32.6,37.3,47.6,51.5,125.8,125.9,128.5,128.6,134.6,135.6,176.0$.

Methyl $\left(2 S^{*}, 3 S^{*}\right)$-3-Phenyl-1,2,3,4-tetrahydronaphthalene-2-carboxylate (3c) (Table 2, entry 2).

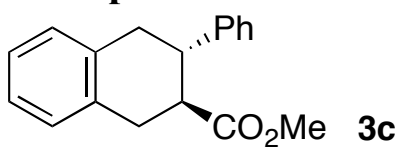

The general procedure was followed with $1 \mathrm{a}(126 \mathrm{mg}, 0.50 \mathrm{mmol})$ and methyl cinnamate $(\mathbf{2 c})(101 \mathrm{mg}$, $0.62 \mathrm{mmol})$. The crude product was purified with MPLC (EtOAc/hexane = 1/2) to give 3c $(105 \mathrm{mg}, 79 \%$ yield) as a colorless solid: mp $84{ }^{\circ} \mathrm{C} ;{ }^{1} \mathrm{H} \mathrm{NMR}\left(400 \mathrm{MHz}, \mathrm{CDCl}_{3}, \mathrm{TMS}\right) \delta$ 2.97-3.29 (m, 6H), 3.43 (s, 3H), 7.07-7.33 (9H); ${ }^{13} \mathrm{C}\left\{{ }^{1} \mathrm{H}\right\} \mathrm{NMR}\left(100 \mathrm{MHz}, \mathrm{CDCl}_{3}\right) \delta 33.1,37.3,43.6,46.9,51.4,126.06,126.11,126.7$, 127.4, 128.5, 128.6, 134.2, 135.5, 143.4, 175.0; IR (neat) 3028, 2919, 1736, $1169 \mathrm{~cm}^{-1}$; Anal. Calcd for $\mathrm{C}_{18} \mathrm{H}_{18} \mathrm{O}_{2}$ : C, 81.17; H, 6.81. Found: C, 81.06; H, 6.83. 
Methyl 2-Methyl-1,2,3,4-tetrahydronaphthalene-2-carboxylate (3d) [117750-14-8] (Table 2, entry 3). ${ }^{8}$

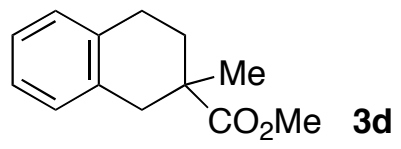

The general procedure was followed with $1 \mathrm{a}(125 \mathrm{mg}, 0.50 \mathrm{mmol})$ and methyl methacrylate $(2 \mathrm{~d})(60 \mathrm{mg}$, $0.60 \mathrm{mmol}$ ). The crude product was purified with MPLC (EtOAc/hexane = 1/3) to give 3c $(48 \mathrm{mg}, 47 \%$ yield) as yellow oil: ${ }^{1} \mathrm{H}$ NMR $\left(400 \mathrm{MHz}, \mathrm{CDCl}_{3}\right.$, TMS) $\delta 1.27(\mathrm{~s}, 3 \mathrm{H}), 1.77$ (dt, J=13.4, $\left.6.9 \mathrm{~Hz}, 1 \mathrm{H}\right), 2.15$ (ddt, $J=1.0,13.4,6.6 \mathrm{~Hz}, 1 \mathrm{H}), 2.65(\mathrm{~d}, J=16.4 \mathrm{~Hz}, 1 \mathrm{H}), 2.81(\mathrm{t}, J=6.7 \mathrm{~Hz}, 2 \mathrm{H}), 3.23(\mathrm{~d}, J=16.4 \mathrm{~Hz}, 1 \mathrm{H})$, 3.65 (s, 3H), 7.00-7.11 (m, 4H); ${ }^{13} \mathrm{C}\left\{{ }^{1} \mathrm{H}\right\}$ NMR (100 MHz, $\left.\mathrm{CDCl}_{3}\right) \delta$ 24.1, 26.2, 31.8, 38.6, 41.7, 51.9, $125.72,125.74,128.7,129.2,134.8,135.0,177.8$.

$\left(2 S^{*}, 3 S^{*}\right)-2-A c e t y l-3-p h e n y l-1,2,3,4-$ tetrahydronaphthalene (3e) [857802-75-6] (Table 2, entry 4). ${ }^{9}$

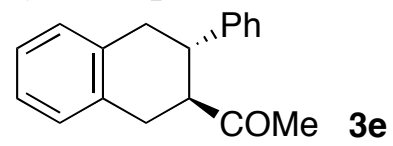

The general procedure was followed with $\mathbf{1 a}(128 \mathrm{mg}, 0.51 \mathrm{mmol})$ and benzalacetone $(\mathbf{2 e})(89 \mathrm{mg}, 0.61$ mmol). The resulting reaction mixture contained two diastereomers, trans- and cis-3e, in the ratio 93:7 (by ${ }^{1} \mathrm{H}$ NMR analysis). The crude product was purified with a flash column chromatography $($ EtOAc/hexane $=$ 1/10) to give pure trans-3e (90 mg, 71\% yield) as a colorless solid: $\mathrm{mp} 74{ }^{\circ} \mathrm{C} ;{ }^{1} \mathrm{H} \mathrm{NMR}\left(400 \mathrm{MHz}, \mathrm{CDCl}_{3}\right.$, TMS) $\delta 1.89(\mathrm{~s}, 3 \mathrm{H}), 2.96-3.26(\mathrm{~m}, 6 \mathrm{H}), 7.06-7.35(9 \mathrm{H}) ;{ }^{13} \mathrm{C}\left\{{ }^{1} \mathrm{H}\right\} \mathrm{NMR}\left(100 \mathrm{MHz}, \mathrm{CDCl}_{3}\right) \delta 30.0,32.6$, $37.6,43.4,53.8,126.09,126.14,126.8,127.4,128.57,128.59,128.7,134.4,135.7,143.7,211.2$; IR (neat) 3026, 2915, $1710 \mathrm{~cm}^{-1}$; Anal. Calcd for $\mathrm{C}_{18} \mathrm{H}_{18} \mathrm{O}: \mathrm{C}, 86.36$; H, 7.25. Found: C, 86.23; H, 7.29.

\section{1,2,3,4-Tetrahydronaphthalene-2-carbonitrile (3f) [51849-33-3] (Table 2, entry 5). ${ }^{6}$}

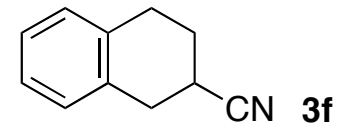

The general procedure except for reaction temperature was followed with $\mathbf{1 a}(126 \mathrm{mg}, 0.50 \mathrm{mmol})$ and acrylonitrile (2e) $(34 \mathrm{mg}, 0.63 \mathrm{mmol})$. The reaction was conducted at $140{ }^{\circ} \mathrm{C}$. The crude product was purified with MPLC $($ EtOAc/hexane $=1 / 5)$ to give $\mathbf{3 f}(33 \mathrm{mg}, 42 \%$ yield $)$ as a colorless solid: ${ }^{1} \mathrm{H}$ NMR (400 $\left.\mathrm{MHz}, \mathrm{CDCl}_{3}, \mathrm{TMS}\right) \delta 2.05$ (ddt, $\left.J=5.7,13.3,9.0 \mathrm{~Hz}, 1 \mathrm{H}\right), 2.16-2.24(\mathrm{~m}, 1 \mathrm{H}), 2.85$ (ddd, $J=6.1,8.4,17.1$ $\mathrm{Hz}, 1 \mathrm{H}), 2.95-3.18(\mathrm{~m}, 4 \mathrm{H}), 7.05-7.19(\mathrm{~m}, 4 \mathrm{H}) ;{ }^{13} \mathrm{C}\left\{{ }^{1} \mathrm{H}\right\} \mathrm{NMR}\left(100 \mathrm{MHz}, \mathrm{CDCl}_{3}\right) \delta 25.5,26.3,27.0,32.3$, $122.0,126.3,126.7,128.9,129.1,132.2,134.5$.

\section{2-Phenyl-1,2,3,4-tetrahydronaphthalene (3g) [29422-13-7] (Table 2, entry 6). ${ }^{10}$}

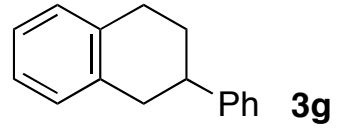

The general procedure was followed with $\mathbf{1 a}(127 \mathrm{mg}, 0.50 \mathrm{mmol})$ and styrene $(\mathbf{2 g})(62 \mathrm{mg}, 0.60 \mathrm{mmol})$. The crude product was purified with a flash column chromatography (hexane) to give $\mathbf{3 g}$ (65 mg, 62\% yield) 
as colorless oil: ${ }^{1} \mathrm{H}$ NMR (400 MHz, $\mathrm{CDCl}_{3}$, TMS) $\delta 1.27(\mathrm{~s}, 3 \mathrm{H}), 1.77(\mathrm{dt}, J=13.4,6.9 \mathrm{~Hz}, 1 \mathrm{H}), 2.15$ (ddt, $J=1.0,13.4,6.6 \mathrm{~Hz}, 1 \mathrm{H}), 2.65(\mathrm{~d}, J=16.4 \mathrm{~Hz}, 1 \mathrm{H}), 2.81(\mathrm{t}, J=6.7 \mathrm{~Hz}, 2 \mathrm{H}), 3.23(\mathrm{~d}, J=16.4 \mathrm{~Hz}, 1 \mathrm{H}), 3.65$ (s, 3H), 7.00-7.11 (m, 4H); ${ }^{13} \mathrm{C}\left\{{ }^{1} \mathrm{H}\right\}$ NMR $\left(100 \mathrm{MHz}, \mathrm{CDCl}_{3}\right) \delta$ 24.1, 26.2, 31.8, 38.6, 41.7, 51.9, 125.72, $125.74,128.7,129.2,134.8,135.0,177.8$.

Reaction of $1 \mathrm{~b}$ with 2a to Give a Mixture of Methyl 6-Methoxy-1,2,3,4-tetrahydronaphthalene-2carboxylate [2473-19-0] $(3 \mathrm{~h})$ and 7-Methoxy-1,2,3,4-tetrahydronaphthalene-2-carboxylate [65844-56-6] (3h') (Table 3, entry 1). ${ }^{11}$

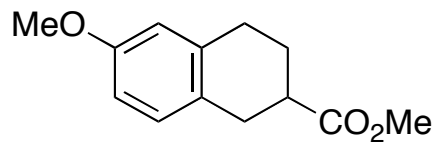

3h

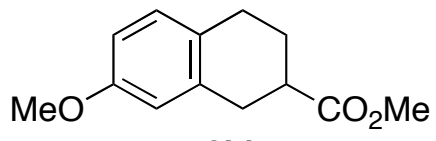

3h'

The general procedure was followed with $1 \mathbf{b}(141 \mathrm{mg}, 0.50 \mathrm{mmol})$ and $2 \mathbf{a}(52 \mathrm{mg}, 0.61 \mathrm{mmol})$. The resulting reaction mixture contained the two regioisomers in the ratio 60:40. It is uncertain which is the major product, $\mathbf{3 h}$ or $\mathbf{3 h}$ '. The crude product was purified with MPLC $($ EtOAc/hexane $=1 / 2)$ to give a mixture of $\mathbf{3 h}$ and $\mathbf{3 h}$ ' (46 mg, 42\% yield) as colorless oil: ${ }^{1} \mathrm{H}$ NMR (400 MHz, $\left.\mathrm{CDCl}_{3}, \mathrm{TMS}\right) \delta 1.78-1.90$ $(\mathrm{m}, 1 \mathrm{H}), 2.15-2.23(\mathrm{~m}, 1 \mathrm{H}), 2.67-3.01(\mathrm{~m}, 5 \mathrm{H}), 3.72(\mathrm{~s}, 3 \mathrm{H}), 3.77(\mathrm{~s}, 3 \mathrm{H}), 6.63(\mathrm{dd}, J=2.7,6.4 \mathrm{~Hz}, 1 \mathrm{H})$, $6.70(\mathrm{dd}, J=2.7,8.4 \mathrm{~Hz}, 1 \mathrm{H}), 7.00(\mathrm{dd}, J=6.6,8.4 \mathrm{~Hz}, 1 \mathrm{H}) ;{ }^{13} \mathrm{C}\left\{{ }^{1} \mathrm{H}\right\} \mathrm{NMR}\left(100 \mathrm{MHz}, \mathrm{CDCl}_{3}\right) \delta 25.8$ (26.2), 28.8 (27.7), 30.9 (31.9), 40.2 (39.9), 51.74 (51.76), 55.2, 112.2 (112.3), 113.4 (113.6), 127.0 (127.8), 129.9 (129.7), 136.8 (135.9), 157.7 (157.6), 175.90 (175.87) (Chemical shifts in parentheses might be assigned to the minor regioisomer.).

The ratio of the two isomers was determined with the ${ }^{1} \mathrm{H}$ NMR analysis in the presence of a shift reagent, $\mathrm{Eu}(\mathrm{fod})_{3}$. The singlet peak at $\delta 3.77$, which was assigned to methoxy on the aromatic ring, was completely separated to two singlets. The integration ratio of the two separated peaks was 60:40.

Reaction of 1c with 2a to Give a Mixture of Methyl 6-Methyl-1,2,3,4-tetrahydronaphthalene-2carboxylate [54730-78-8] (3i) and 7-Methyl-1,2,3,4-tetrahydronaphthalene-2-carboxylate [857802-80-3] (3i') (Table 3, entry 2). ${ }^{9}$

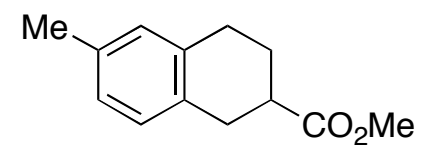

3i

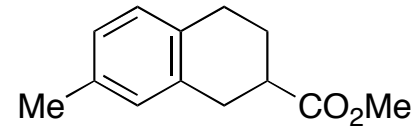

$3 \mathbf{i}^{\prime}$

The general procedure was followed with $1 \mathbf{c}(135 \mathrm{mg}, 0.51 \mathrm{mmol})$ and $2 \mathbf{a}(53 \mathrm{mg}, 0.61 \mathrm{mmol})$. The resulting reaction mixture contained the two regioisomers in the ratio 54:46. It is uncertain which is the major product, 3i or 3i'. The crude product was purified with MPLC (EtOAc/hexane $=1 / 10)$ to give a mixture of 3i and 3i' (75 mg, 72\% yield) as colorless oil: ${ }^{1} \mathrm{H}$ NMR (400 MHz, $\mathrm{CDCl}_{3}$, TMS) $\delta 1.78-1.89$ (m, $1 \mathrm{H}), 2.15-2.24(\mathrm{~m}, 1 \mathrm{H}), 2.28(\mathrm{~s}, 3 \mathrm{H}), 2.68-3.03(\mathrm{~m}, 5 \mathrm{H}), 3.72(\mathrm{~s}, 3 \mathrm{H}), 6.88-7.01(\mathrm{~m}, 3 \mathrm{H}) ;{ }^{13} \mathrm{C}\left\{{ }^{1} \mathrm{H}\right\} \mathrm{NMR}$ $\left(100 \mathrm{MHz}, \mathrm{CDCl}_{3}\right) \delta$ 20.9, 25.9 (26.1), 28.5 (28.1), 31.3 (31.6), 40.0 (40.1), 51.7, 126.6 (126.8), 128.9 (128.7), 129.4 (129.6), 131.8 (132.5), 135.38 (135.26), 135.44 (134.6), 175.9 (Chemical shifts in parentheses 
might be assigned to the minor regioisomer.); IR (neat) 2949, 2925, 1738, 1730, $1170 \mathrm{~cm}^{-1}$; Anal. Calcd for $\mathrm{C}_{13} \mathrm{H}_{16} \mathrm{O}_{3}$ : C, 76.44; H, 7.90. Found: C, 76.31; H, 7.85.

The ratio of the two isomers was determined with the ${ }^{1} \mathrm{H}$ NMR analysis in the presence of a shift reagent, $\mathrm{Eu}(\mathrm{fod})_{3}$. The singlet peak at $\delta 2.28$, which was assigned to methyl on the aromatic ring, was completely separated to two singlets. The integration ratio of the two separated peaks was 54:46.

Reaction of 1d with 2a to Give a Mixture of Methyl 5-Phenyl-1,2,3,4-tetrahydronaphthalene-2carboxylate (3j) and 8-Phenyl-1,2,3,4-tetrahydronaphthalene-2-carboxylate (3j') (Table 3, entry 3).

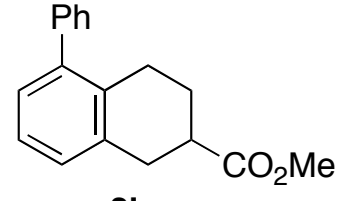

3j

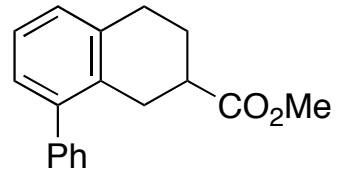

$3 \mathbf{j}^{\prime}$

The general procedure was followed with $1 \mathbf{d}(164 \mathrm{mg}, 0.50 \mathrm{mmol})$ and $\mathbf{2 a}(52 \mathrm{mg}, 0.61 \mathrm{mmol})$. The resulting reaction mixture contained the two regioisomers in the ratio 55:45. It is uncertain which is the major product, $\mathbf{3 j}$ or $\mathbf{3 j}$ '. The crude product was purified with MPLC (EtOAc/hexane $=1 / 5)$ to give a mixture of $\mathbf{3 j}$ and 3j' (123 mg, 92\% yield) as yellow oil: ${ }^{1} \mathrm{H}$ NMR (400 MHz, $\left.\mathrm{CDCl}_{3}, \mathrm{TMS}\right) \delta 1.76$ (ddt, $J=$ $11.2,13.0,8.2 \mathrm{~Hz}, 0.45 \mathrm{H}), 1.90$ (ddt, $J=6.2,12.9,10.8 \mathrm{~Hz}, 0.55 \mathrm{H}), 2.09-2.21(\mathrm{~m}, 1 \mathrm{H}), 2.59-3.16(\mathrm{~m}, 5 \mathrm{H})$, $3.64(\mathrm{~s}, 1.65 \mathrm{H}), 3.71(\mathrm{~s}, 1.35 \mathrm{H}), 7.04-7.21(\mathrm{~m}, 3 \mathrm{H}), 7.26-7.43(\mathrm{~m}, 5 \mathrm{H}) ;{ }^{13} \mathrm{C}\left\{{ }^{1} \mathrm{H}\right\} \mathrm{NMR}\left(100 \mathrm{MHz}, \mathrm{CDCl}_{3}\right) \delta$ $25.7,26.1,27.3,29.0,30.4,32.1,39.6,40.3,51.6,51.7,125.58,125.62,126.79,126.81,127.5,128.01$, $128.06,128.09,128.3,129.1,132.5,133.4,135.2,136.0,141.5,141.6,141.9,142.2,175.85,175.94$; IR (neat) 2949, 1736, $1169 \mathrm{~cm}^{-1}$; HRMS Calcd for $\mathrm{C}_{18} \mathrm{H}_{18} \mathrm{O}_{2}: 266.1307$. Found: $m / z$ 266.1306.

Reaction of $1 \mathrm{e}$ with 2 a to Give a Mixture of Methyl 6,8-Dimethyl-1,2,3,4-tetrahydronaphthalene-2carboxylate (3k) and 5,7-Dimethyl-1,2,3,4-tetrahydronaphthalene-2-carboxylate (3k') (Table 3, entry 4).
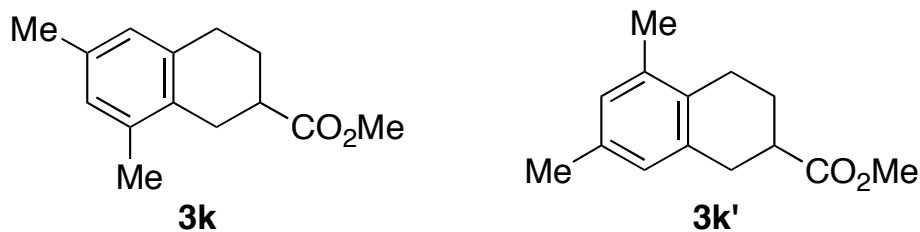

The general procedure was followed with 1 e $(142 \mathrm{mg}, 0.51 \mathrm{mmol})$ and $\mathbf{2 a}(52 \mathrm{mg}, 0.60 \mathrm{mmol})$. The resulting reaction mixture contained the two regioisomers in the ratio 60:40. It is uncertain which is the major product, 3k or 3k'. The crude product was purified with MPLC $($ EtOAc/hexane $=1 / 5)$ to give a mixture of 3k and 3k' (57 mg, 51\% yield) as colorless oil: ${ }^{1} \mathrm{H}$ NMR (400 MHz, $\mathrm{CDCl}_{3}$, TMS) $\delta 1.73-1.90$ $(\mathrm{m}, 1 \mathrm{H}), 2.14-2.30(\mathrm{~m}, 1 \mathrm{H}), 2.18(\mathrm{~s}, 1.2 \mathrm{H}), 2.20(\mathrm{~s}, 1.8 \mathrm{H}), 2.25(\mathrm{~s}, 3 \mathrm{H}), 2.52-2.99(\mathrm{~m}, 5 \mathrm{H}), 3.72(\mathrm{~s}, 1.2 \mathrm{H})$, $3.74(\mathrm{~s}, 1.8 \mathrm{H}), 6.77(\mathrm{~s}, 1 \mathrm{H}), 6.83(\mathrm{~s}, 1 \mathrm{H}) ;{ }^{13} \mathrm{C}\left\{{ }^{1} \mathrm{H}\right\} \mathrm{NMR}\left(100 \mathrm{MHz}, \mathrm{CDCl}_{3}\right) \delta 19.42(19.36), 20.8,25.66$ (25.74), 28.8 (26.2), 29.1 (32.2), 40.4 (39.7), 51.75 (51.71), 127.1 (127.3), 128.3 (128.4), 130.3 (131.0), 135.0 (134.7), 135.5 (134.9), 136.5 (136.3), 176.2 (176.0) (Chemical shifts in parentheses might be assigned 
to the minor regioisomer.); IR (neat) $2949,1738,1729,1174 \mathrm{~cm}^{-1}$; Anal. Calcd for $\mathrm{C}_{14} \mathrm{H}_{18} \mathrm{O}_{2}: \mathrm{C}, 77.03 ; \mathrm{H}$, 8.31. Found: C, 77.00; H, 8.28.

The ratio of the two isomers was determined with the ${ }^{1} \mathrm{H}$ NMR analysis in the presence of a shift reagent, $\mathrm{Eu}$ (fod) . $_{3}$ The two singlet peaks at $\delta 3.72$ and 3.74, which were assigned to methyl on the ester group, were completely separated to two peaks. The integration ratio of the two separated peaks was 60:40.

\section{References}

(1) Lenihan, B. D.; Shechter, H. J. Org. Chem. 1998, 63, 2072-2085.

(2) Meyers, M. J.; Sun, J.; Carson, K. E.; Marriner, G. A.; Katzenellenbogen, B. S.; Katzenellenbogen, J. A. J. Med. Chem. 2001, 44, 4230-4251.

(3) Tatsuno, Y.; Yoshida, T.; Otsuka, S. Inorg. Synth. 1979, 19, 220-223.

(4) Tatsuno, Y.; Yoshida, T.; Otsuka, S. Inorg. Synth. 1990, 28, 342-345.

(5) Yoshida, H.; Nakano, S.; Yamaryo, Y.; Ohshita, J.; Kunai, A. Org. Lett. 2006, 8, 4157-4159.

(6) Mills, R. J.; Taylor, N. J.; Snieckus, V. J. Org. Chem. 1989, 54, 4372-4385.

(7) Inaba, S.-i.; Wehmeyer, R. M.; Forkner, M. W.; Rieke, R. D. J. Org. Chem. 1988, 53, 339-344.

(8) Eru, E.; Hawkes, G. E.; Utley, J. H. P.; Wyatt, P. B. Tetrahedron 1995, 51, 3033-3044.

(9) Kuwano, R.; Shige, T. Chem. Lett. 2005, 34, 728-729.

(10) Jung, M. E.; Mossman, A. B.; Lyster, M. A. J. Org. Chem. 1978, 43, 3698-3701.

(11) Ito, Y.; Nakatsuka, M.; Saegusa, T. J. Am. Chem. Soc. 1982, 104, 7609-7622. 


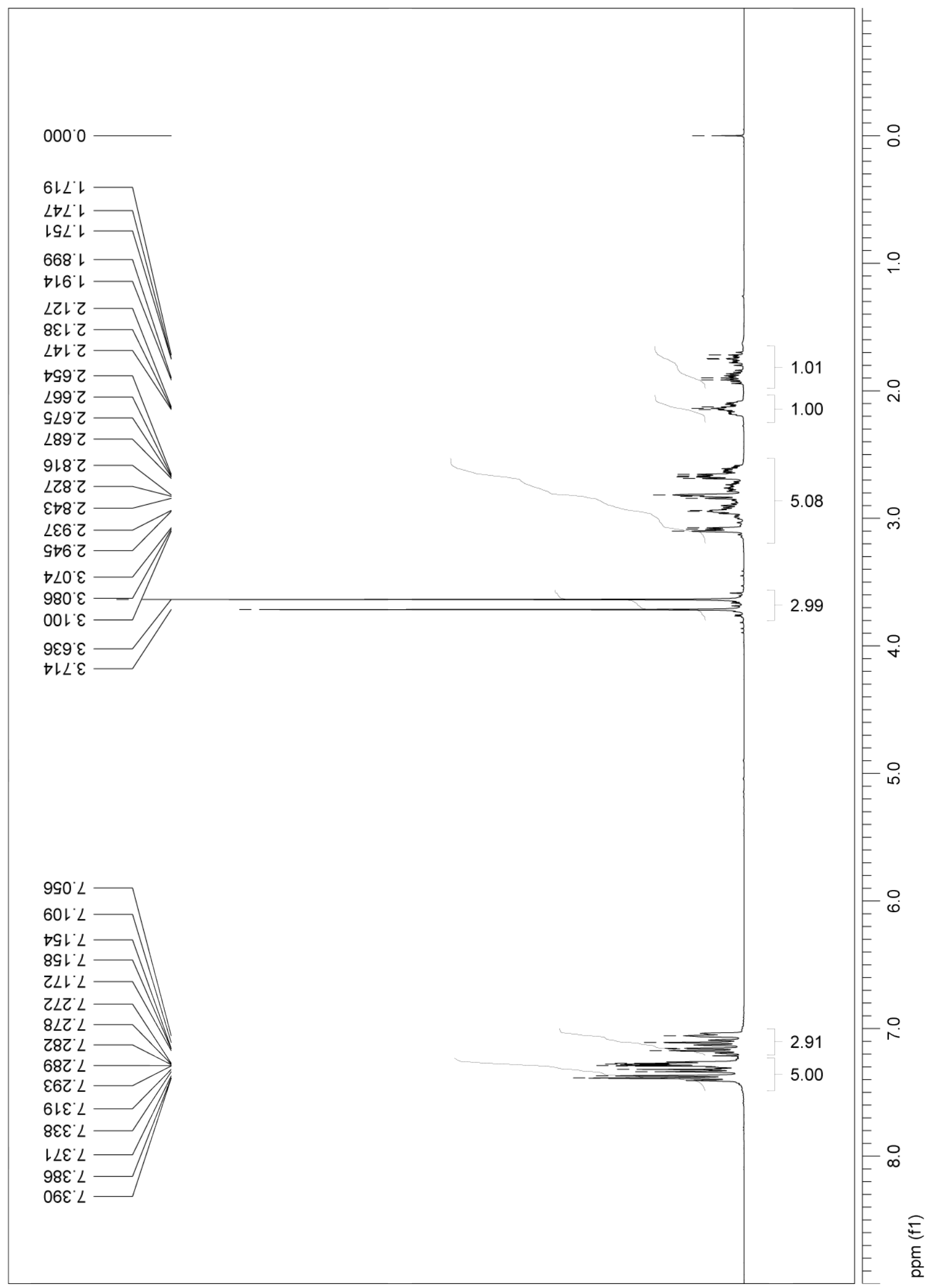

Figure S-1. $\quad{ }^{1} \mathrm{H}$ NMR spectrum of the mixture of $\mathbf{3 j}$ and $\mathbf{3 j}$ '. 


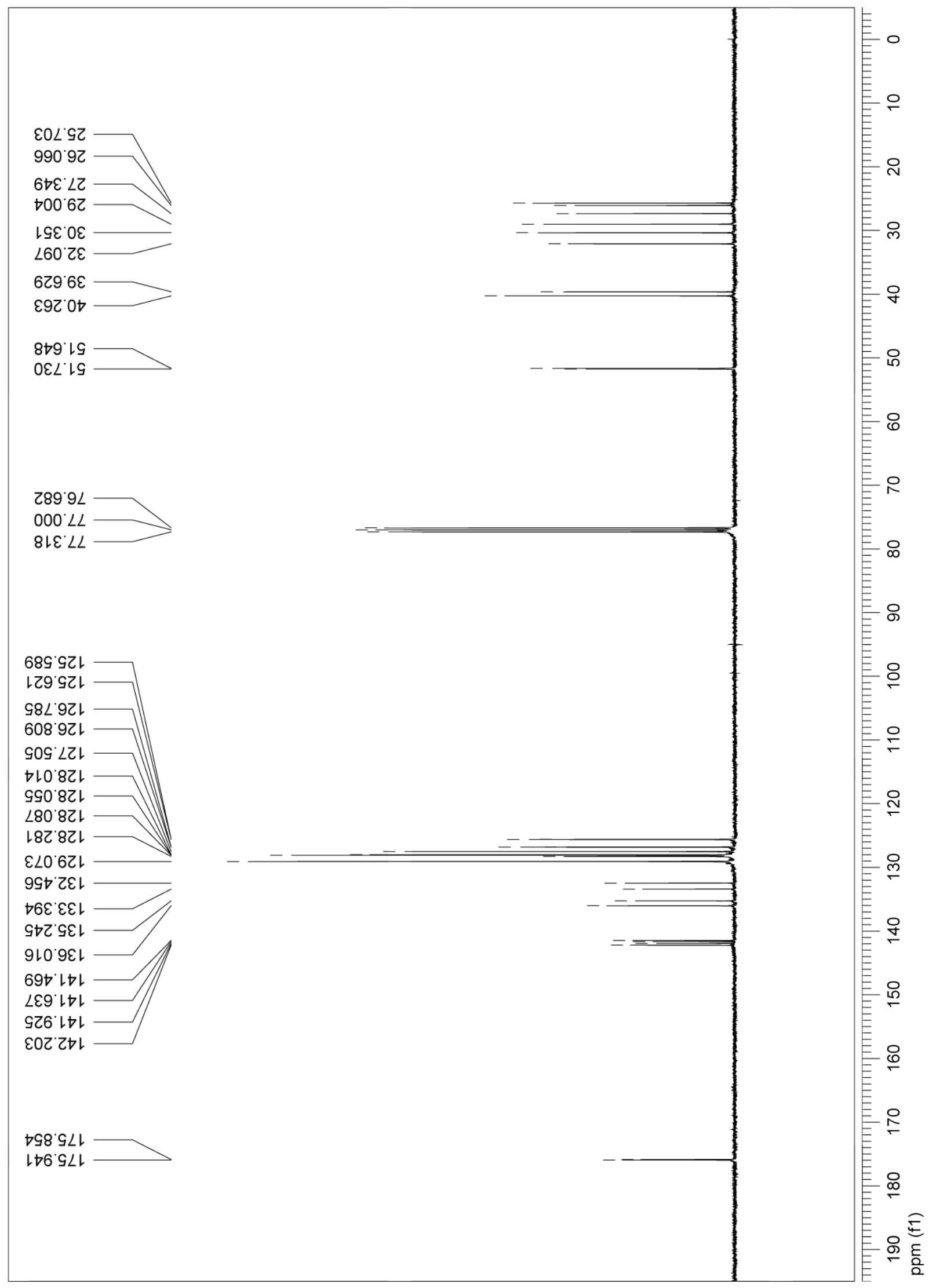

Figure S-2. ${ }^{13} \mathrm{C}\left\{{ }^{1} \mathrm{H}\right\}$ NMR spectrum of the mixture of $\mathbf{3 j}$ and $\mathbf{3 j}$ '. 